PROCEEDINGS OF THE

AMERICAN MATHEMATICAL SOCIETY

Volume 139, Number 7, July 2011, Pages 2565-2569

S 0002-9939(2010)10669-X

Article electronically published on December 7, 2010

\title{
A REMARK ON THE ISOTROPY CONSTANT OF POLYTOPES
}

\author{
DAVID ALONSO-GUTIÉRREZ
}

(Communicated by Marius Junge)

\begin{abstract}
It is known that the isotropy constant of any symmetric polytope with $2 N$ vertices is bounded by $C \log N$. We give a different proof of this result, which shows that the same estimate is true when the polytope is nonsymmetric with $N$ vertices. We also make a remark on how an estimate of the isotropy constant of a symmetric polytope with $2 N$ facets of the order of $\sqrt{\log \frac{N}{n}}$, which can be easily deduced from known results, is also true for non-symmetric polytopes with $N$ facets.
\end{abstract}

\section{INTRODUCTION AND NOTATION}

A convex body $K \subset \mathbb{R}^{n}$ is a compact convex set with non-empty interior. A convex body is said to be in isotropic position if it has volume 1 and satisfies the following two conditions:

- $\int_{K} x d x=0$ (center of mass at 0$)$,

- $\int_{K}\langle x, \theta\rangle^{2} d x=L_{K}^{2} \quad \forall \theta \in S^{n-1}$,

where $L_{K}$ is a constant independent of $\theta$, which is called the isotropy constant of $K$. Here $\langle\cdot, \cdot\rangle$ denotes the standard scalar product in $\mathbb{R}^{n}$.

It is well known that for every convex body $K \subset \mathbb{R}^{n}$ there exists an affine map $a+T$ such that $a+T K$ is isotropic. Furthermore, $K$ and $T K$ are both isotropic if and only if $T$ is an orthogonal transformation and then both $K$ and $T K$ have the same isotropy constant. Hence, we can define the isotropy constant for any convex body as the isotropy constant of its isotropic image. This verifies that

$$
n L_{K}^{2}=\min _{\substack{T \in G L(n) \\ a \in \mathbb{R}^{n}}}\left\{\frac{1}{|T K|^{1+\frac{2}{n}}} \int_{a+T K}|x|^{2} d x\right\},
$$

so the isotropic position is the one that minimizes the quantity in the right hand side of the equation among all the affine images of a convex body.

It is conjectured that there exists an absolute constant $C$ such that for every convex body $K, L_{K} \leq C$. This conjecture has been verified for several classes of convex bodies such as unconditional bodies $\mathrm{MP}$, zonoids Ba and others (e.g., KMP $)$. However, the best known general upper bound is $L_{K} \leq c n^{\frac{1}{4}}$, [K], which improves the earlier estimate $L_{K} \leq c n^{\frac{1}{4}} \log n$ given by Bourgain (see [Bo]) for symmetric convex bodies and extended by Paouris $[\mathrm{Pa}$ for non-symmetric bodies.

Received by the editors June 24, 2010.

2010 Mathematics Subject Classification. Primary 52B99.

The author was supported by MCYT grants (Spain) MTM2007-61446; DGA E-64.

(C) 2010 American Mathematical Society 
Since every convex body can be approximated by polytopes, the conjecture is true if and only if it is true for any polytope in any dimension. M. Junge proved in $[\mathrm{Ju}$ ] that every centrally symmetric polytope with $2 N$ vertices has its isotropy constant bounded by $C \log N$, and E. Milman gave in Mi another proof of this fact. Their proofs use results in the local theory of normed spaces. Our purpose is to give here a simpler proof which is also valid for non-symmetric polytopes. We will prove

Theorem 1.1. Let $K \subset \mathbb{R}^{n}$ be a polytope with $N$ vertices. There exists an absolute constant $C$ such that $L_{K} \leq C \log N$.

If the number of vertices is large enough, this estimate is better than $L_{K} \leq$ $C \sqrt{\frac{N}{n}}$, proved in ABBW], which is better when the number of vertices is small.

We will also prove an estimate of the isotropy constant of polytopes involving the number of facets instead of the number of vertices. The result is the following:

Theorem 1.2. Let $K \subseteq \mathbb{R}^{n}$ be a polytope with $N$ facets. Then its isotropy constant satisfies

$$
L_{K} \leq C \sqrt{\log \frac{N}{n}} .
$$

In the next section we will give the proofs of these results. We will use the notation $h_{K}(\cdot)$ for the support function of $K, h_{K}(u)=\sup _{x \in K}\langle x, u\rangle, R(K)$ for its circumradius $R(K)=\max \{|x|: x \in K\}$ and $r(K)$ for its inradius $r(K)=\max \{r \geq$ $\left.0: r B_{2}^{n} \subseteq K\right\}$, where $B_{2}^{n}$ is the $n$-dimensional Euclidean ball.

\section{Proof of the Results}

In this section we are going to prove the aforementioned results.

Proof of Theorem 1.1. First of all notice that if $K \subset \mathbb{R}^{n}$ is isotropic, as a consequence of Borell's lemma, for any $\theta \in S^{n-1}$ we have $\|\langle\cdot, \theta\rangle\|_{\psi_{1}} \leq C L_{K}$, where

$$
\|f\|_{\psi_{\alpha}}=\inf \left\{t>0: \int_{K} e^{\left(\frac{|f(x)|}{t}\right)^{\alpha}} d x \leq 2\right\}
$$

As a consequence of this, $\int_{K} \max _{i=1, \ldots, N}\left|\left\langle x, \theta_{i}\right\rangle\right| d x \leq C L_{K}(\log N)$ for any $\theta_{1}, \ldots$, $\theta_{N} \in S^{n-1}$.

Let $K$ be a polytope with vertices $P_{1}, \ldots, P_{N}$ in isotropic position. By the definition of isotropy, for any $T \in G L(n)$ that is symmetric and positive definite,

$$
\begin{aligned}
L_{K}^{2} & =\frac{1}{\operatorname{tr} T} \int_{K}\langle T x, x\rangle d x=\frac{1}{\operatorname{tr} T} \int_{K}|\langle T x, x\rangle| d x \leq \frac{1}{\operatorname{tr} T} \int_{K} \max _{y \in K}|\langle T y, x\rangle| d x \\
& =\frac{1}{\operatorname{tr} T} \int_{K} \max _{i=1, \ldots, N}\left|\left\langle T P_{i}, x\right\rangle\right| d x \leq \frac{\max _{i=1, \ldots, N}\left|T P_{i}\right|}{\operatorname{tr} T} \int_{K} \max _{i=1, \ldots, N}\left|\left\langle\frac{T P_{i}}{\left|T P_{i}\right|}, x\right\rangle\right| d x \\
& \leq \frac{C L_{K} \log N \max _{i=1, \ldots, N}\left|T P_{i}\right|}{\operatorname{tr} T}
\end{aligned}
$$

since $K$ is isotropic.

Thus, for any such $T$,

$$
\begin{aligned}
L_{K} & \leq \frac{C \log N \max _{i=1, \ldots, N}\left|T P_{i}\right|}{\operatorname{tr} T} \leq \frac{C \log N \max _{i=1, \ldots, N}\left|T P_{i}\right|}{n(\operatorname{det} T)^{\frac{1}{n}}} \\
& =\frac{C \log N \max _{i=1, \ldots, N}\left|T P_{i}\right|}{n|T K|^{\frac{1}{n}}}
\end{aligned}
$$


Now take $T$ such that $a+T K$ is in Lowner's position; i.e., the Euclidean ball is the minimum volume ellipsoid containing $a+T K$. Then for any $P_{i},\left|T P_{i}\right| \leq$ $\left|a+T P_{i}\right|+|a| \leq 2$, since $a$ and $a+T P_{i}$ are the centroid and a vertex of $a+T K$, respectively, and consequently they are contained in the Euclidean ball. If $K$ is a symmetric polytope, then it is known that $a=0, r(T K) \geq \frac{1}{\sqrt{n}}$ and consequently $|T K|^{\frac{1}{n}} \geq \frac{c}{n}$ and we obtain the desired estimate $L_{K} \leq C \log N$. However, if $K$ is not symmetric, it is not true that $r(a+T K) \geq \frac{1}{\sqrt{n}}$ (the correct estimate in this case is $\left.r(a+T K) \geq \frac{1}{n}\right)$.

Nevertheless, as a consequence of a result proved in [D], it is still true that $|T K|^{\frac{1}{n}}=|a+T K|^{\frac{1}{n}} \geq \frac{c}{n}$ and consequently $L_{K} \leq C \log N$ also in the non-symmetric case. Indeed, we start by recalling the following well known result, which gives a characterization of convex bodies $K$ in Lowner's or John's position (with the Euclidean ball as the maximum volume ellipsoid contained in $K$ ).

Theorem 2.1 $([\mathrm{J}])$. Let $K \subset \mathbb{R}^{n}$ be a convex body. $K$ is in Lowner's position (resp. John's position) if and only if $K \subset B_{2}^{n}$ (resp. $B_{2}^{n} \subseteq K$ ) and there exist some vectors $\left\{u_{i}\right\}_{i=1}^{m} \in \partial K \cap S^{n-1}$ and some positive numbers $\left\{c_{i}\right\}_{i=1}^{m}$ satisfying

- $\sum_{i=1}^{m} c_{i} u_{i}=0$,

- $I_{n}=\sum_{i=1}^{m} c_{i} u_{i} \otimes u_{i}$,

where $u \otimes u$ denotes the rank 1 operator which maps $x$ to $\langle x, u\rangle u$.

A system $\left\{\left(u_{i}, \lambda_{i}\right)\right\}_{i=1}^{m}$ of vectors $u_{i} \in \mathbb{R}^{n}$ and positive weights $\lambda_{i}$ such that $\sum_{i=1}^{m} \lambda_{i}=1$ is called isotropic if it satisfies the following two conditions:

- $\sum_{i=1}^{m} \lambda_{i} u_{i}=0$,

- $\sum_{i=1} \lambda_{i} u_{i} \otimes u_{i}=L^{2}\left|\operatorname{conv}\left\{u_{i}\right\}_{i=1}^{m}\right|^{\frac{2}{n}} I_{n}$.

With this definition it is clear that if $K$ is in Lowner's position and $\left\{u_{i}\right\}_{i=1}^{m}$ and $\left\{c_{i}\right\}_{i=1}^{m}$ are the vectors and numbers defined in Theorem 2.1, then the system $\left\{\left(u_{i}, \frac{c_{i}}{n}\right)\right\}$ is isotropic with $L^{2}\left|\operatorname{conv}\left\{u_{i}\right\}_{i=1}^{m}\right|^{\frac{2}{n}}=\frac{1}{n}$. (Notice that, taking traces in the decomposition of the identity, necessarily $\sum_{i=1}^{m} c_{i}=n$.) In $[\mathrm{D}$, it is proved for any isotropic system of vectors $L \leq C \sqrt{n}$.

Using this estimate we deduce that whenever $K$ is in Lowner's position and the $c_{i}$ 's and $u_{i}$ 's are defined as before,

$$
\frac{1}{n}=L^{2}\left|\operatorname{conv}\left\{u_{i}\right\}_{i=1}^{m}\right|^{\frac{2}{n}} \leq C n|K|^{\frac{2}{n}}
$$

and consequently $|K|^{\frac{1}{n}} \geq \frac{c}{n}$.

Now we will prove the result concerning the number of facets of the polytope.

Proof of Theorem 1.2. Since

$$
n L_{K}^{2}=\min _{\substack{T \in G L(n) \\ a \in \mathbb{R}^{n}}}\left\{\frac{1}{|T K|^{1+\frac{2}{n}}} \int_{a+T K}|x|^{2} d x\right\}
$$

we can assume that $K$ is in John's position and hence

$$
n L_{K}^{2} \leq \frac{1}{|K|^{\frac{2}{n}}} \frac{1}{|K|} \int_{K}|x|^{2} d x
$$


In $\mathrm{BaP}$ it is proved that there exists an absolute constant $c$ such that if $\left\{u_{i}\right\}_{i=1}^{N} \subset \mathbb{R}^{n}$ are vectors of length at most 1 , then

$$
\left|\left\{x \in \mathbb{R}^{n}:\left|\left\langle x, u_{i}\right\rangle\right| \leq 1, i=1, \ldots, N\right\}\right|^{\frac{1}{n}} \geq \frac{c}{\sqrt{1+\log \frac{N}{n}}} .
$$

Thus

$$
|K|^{\frac{1}{n}} \geq \frac{c}{\sqrt{1+\log \frac{N}{n}}}
$$

and combining this with the fact that any symmetric convex body in John's position has its outer radius bounded from above by $\sqrt{n}$, we obtain the desired estimate.

However, in the case that $K$ is in John's position but is not symmetric, it is not true that the outer radius of $K$ is bounded by $\sqrt{n}$. The right estimate in this case is $R(K) \leq n$. Instead of assuming that $K$ is in John's position, we assume that the center of mass of $K$ is at the origin and $K \cap(-K)$ is in John's position. Then there exist some vectors $\left\{u_{i}\right\}_{i=1}^{m} \in \partial(K \cap(-K)) \cap S^{n-1}$ and some positive numbers $\left\{c_{i}\right\}_{i=1}^{m}$ such that $I_{n}=\sum_{i=1}^{m} c_{i} u_{i} \otimes u_{i}$.

Since we have a decomposition of the identity independently from the fact that we take the vector $u_{i}$ or its opposite, we can assume that all the vectors $u_{i} \in \partial K \cap S^{n-1}$. Hence $h_{K}\left(u_{i}\right)=1$ and using the estimate $\frac{1}{|K|} \int_{K}\left\langle x, u_{i}\right\rangle^{2} d x \leq \frac{n h_{K}\left(u_{i}\right)^{2}}{n+2}$ whenever the center of mass of $K$ is at 0 , which is proved in [KLS], we obtain

$$
\begin{aligned}
n L_{K}^{2} & \leq \frac{1}{|K|^{\frac{2}{n}}} \frac{1}{|K|} \int_{K}|x|^{2} d x \leq \frac{1}{|K \cap(-K)|^{\frac{2}{n}}} \sum_{i=1}^{m} c_{i} \frac{1}{|K|} \int_{K}\left\langle x, u_{i}\right\rangle^{2} d x \\
& \leq c \log \frac{N}{n} \sum_{i=1}^{m} c_{i} \frac{n h_{K}\left(u_{i}\right)^{2}}{n+2}=\frac{c n^{2} \log \frac{N}{n}}{n+2} \leq c n \log \frac{N}{n} .
\end{aligned}
$$

Consequently

$$
L_{K} \leq C \sqrt{\log \frac{N}{n}}
$$

Remark 2.2. It seems natural to try to deduce this estimate assuming that $K$ is in John's position also in the non-symmetric case. When one tries to do it, the problem arises of estimating how far from the origin is the centroid of the convex body. If it were true that the center of mass of a convex body in John's position is closer to the origin than $c \sqrt{n}$, then, using the results in [FPS], we could deduce the same estimate for non-symmetric polytopes by considering them in John's position. Unfortunately, an estimate of such type is not known.

\section{REFERENCES}

ABBW. Alonso-Gutiérrez D., Bastero J., Bernués J., Wolff P. On the isotropy constant of projections of polytopes. Journal of Functional Analysis 258 (2010), no. 5, pp. 1452-1465. MR2566308

Ba. Ball K. Normed spaces with a weak Gordon-Lewis property. Springer Lecture Notes in Math., 1470, Springer, Berlin, 1991, pp. 36-47. MR.1126735 (93e:46013)

BaP. Ball K., Pajor A. Convex bodies with few faces. Proceedings of the American Mathematical Society 110 (1990), no. 1, pp. 225-231. MR1019270 (90m:52011)

Bo. Bourgain J. On the distribution of polynomials on high-dimensional convex sets, Springer Lecture Notes in Math., 1469, Springer, Berlin, 1991, pp. 127-137. MR.1122617 (92j:52007) 
D. Dar S. On the isotropic constant of non-symmetric convex bodies. Israel J. Math. 97 (1997), pp. 151-156. MR.1441244 (98a:52012)

FPS. Fradelizi M., Paouris G., Schütt C. Simplices in the Euclidean ball. To appear in Canad. Math. Bull.

J. John F. Extremum problems with inequalities as subsidiary conditions, Courant Anniversary Volume, Interscience, New York, 1948, pp. 187-204. MR0030135 (10:719b)

Ju. Junge M., Proportional subspaces of spaces with unconditional basis have good volume properties. Geometric aspects of functional analysis, Oper. Theory Adv. Appl., 77, pp. 121-129, Birkhäuser, Basel, 1995. MR.1353455 (96m:52011)

KLS. Kannan R., Lovász L., Simonovits M. Isoperimetric problems for convex bodies and a localization lemma. Discrete Comput. Geom. 13 (1995), pp. 541-559. MR.1318794 (96e:52018)

K. Klartag, B. On convex perturbations with a bounded isotropic constant. Geom. Funct. Anal. 16 (2006), no. 6, pp. 1274-1290. MR2276540 (2007i:52005)

KMP. König H., Meyer M., Pajor A. The isotropy constants of the Schatten classes are bounded. Math. Ann. 312 (1998) no. 4. pp. 773-783. MR1660231 (99j:52003)

Mi. Milman E. Dual mixed volumes and the slicing problem, Adv. Math. 207 (2006), pp. 566598. MR 2271017 (2007i:52006)

MP. Milman V., Pajor A. Isotropic position and inertia ellipsoids and zonoids of the unit ball of a normed n-dimensional space, GAFA Seminar 87-88, Springer Lecture Notes in Math., 1376, Springer, Berlin, 1989, pp. 64-104. MR1008717 (90g:52003)

Pa. Paouris G. On the isotropic constant of non-symmetric convex bodies, Geometric Aspects of Functional Analysis, Lecture Notes in Mathematics, 1745, Springer, Berlin, 2000, pp. 239-243. MR.1796722 (2002b:52013)

Departmento de Matemáticas, Facultad de Ciencias, Universidad de Zaragoza, 50009 ZARAGOZA, Spain

E-mail address: daalonso@unizar.es

Current address: The Fields Institute for Research in Mathematical Sciences, 222 College Street, Second Floor, Toronto, Ontario M5T 3J1, Canada 